\title{
Surrogate Markers of Liver Fibrosis in Primary Sclerosing Cholangitis (PSC)
}

\author{
Monika Szydlowska-Jakimiuk ${ }^{1}$, Joanna Raszeja-Wyszomirska*2 \\ ${ }^{1}$ Clinic of Hepatology and Internal Diseases Department of General Surgery, Transplantation And Liver, Warsaw Medical University, Warsaw
}

${ }^{2}$ Liver and Internal Medicine Unit, Department of General, Transplant and Liver Surgery, Medical University of Warsaw, Warsaw, Poland

${ }^{*}$ Correspondence to: Joanna Raszeja-Wyszomirska, Liver and Internal Medicine Unit, Department of General, Transplant and Liver Surgery, Medical University of Warsaw, Warsaw, Poland; Tel/Fax: +48 22 5991662,63; E-mail: joanna.raszeja-wyszomirska@wum.edu.pl

Received: August 03, 2017; Accepted: August 16, 2017; Published: August 25, 2017;

\section{Introduction}

Primary Sclerosing Cholangitis (PSC) is a chronic inflammatory cholangiopathy that results in fibrotic strictures and dilations of the intra- and extrahepatic bile ducts. The pathogenesis of PSC has not been fully elucidated, the disease is uncommon, occurs predominantly in young males and has a strong association with Inflammatory Bowel Disease (IBD). There are significant variation in clinical course of PSC associated with age at diagnosis, sex, and ductal and IBD subtypes [1]. There is no medical treatment of proven benefit on survival; most liver-related morbidity and mortality is the results of portal hypertension and chronic liver failure. However, the course of PSC is highly variable, and so far no prognostic markers have been shown to predict outcomes in asymptomatic, early-stage patients.

The prognosis of chronic cholestatic liver disease depends at least in part on the extent of fibrosis in the liver parenchyma [2]. Semiquantitative evaluation of nodular size and fibrotic septal width in respect to hepatic venous pressure gradient (HVPG) were proposed by Laennec based on the original histological description of the cirrhosis [3-6]. HVPG is the gold standard to estimate the severity of portal hypertension in liver cirrhosis. It correlates with structural and functional changes in liver parenchyma and gives valuable prognostic information to stratify the mortality risk [7]. Liver cirrhosis should be regarded as a multistage liver disease [8]; it can be accurately subclassified using quantification of fibrosis with collagen proportionate area (CPA) as the predictor of clinical decompensation [9].

Liver biopsy remains the "gold standard" in evaluation of necroinflammation activity and fibrosis of the liver parenchyma. However, it has limitations due to invasiveness, small tissue samples, patchy distribution of fibrotic areas in parenchyma and inter- and intra-observer error. Moreover, liver biopsy is not appropriate to regularly monitor fibrosis progression or response to treatment [10]. Thus, ultrasound-based shear wave elastography methods enabling liver stiffness measurements (LSM) have been implemented for noninvasive evaluation of fibrosis of the liver, with biopsy reserved for uncertain cases.

The various elastography methods differ with respect to what they do with these displacement data to create an elastogram or elasticity measurement. There are three options for the property to be displayed:
1. Display of displacement without further processing, as in acoustic radiation force impulse (ARFI) imaging. Tissue displacement is associated with shear deformation. The greater the force, the greater the displacement, but stiff tissues are displaced less than soft tissues. ARFI remains the proprietary imaging technology Siemens Virtual Touch ${ }^{\mathrm{Tx}}$, and it is not used for assessment of diffuse liver conditions.

2. Display of tissue strain or strain rate, calculated from the spatial gradient of displacement or velocity,

3. Display of shear wave speed, calculated by using the time varying displacement data to measure the arrival time of a shear wave at various locations. All such methods are grouped under the heading shear wave elastography (SWE), and include transient elastography (TE), point shear wave elastography (pSWE) and multidimensional shear wave elastography (2D-SWE and 3D-SWE).

Shear wave elastography (SWE) is a method that use shear wave speed and includes:

1. Transient elastography (TE, FibroScan, Echosens, France): shear wave elastometry by measurement of the speed of a shear wave that has been generated using a surface impulse,

2. Point shear wave elastography (pSWE): shear wave elastometry at a location by measurement of the speed of a shear wave generated using acoustic radiation force,

3. Multidimensional shear wave elastography (2D-SWE, 3DSWE): quantitative SWE imaging (and elastometry) by measuring the speed of shear waves generated using acoustic radiation force.

The major potential confounding factors (liver inflammation indicated by AST and/or ALT elevation $>5$ times the normal limits, obstructive cholestasis, liver congestion, acute hepatitis and infiltrative liver diseases) should be excluded before performing LSM with SWE, in order to avoid overestimation of liver fibrosis [11].

\section{Ultrasound-based methods}

In chronic liver disease LSM accurately reflects liver fibrosis, which is the major component of increased intrahepatic vascular resistance 
leading to portal hypertension. LSM improves the noninvasive risk stratification of patients with compensated advanced chronic liver disease as a possible surrogate for portal hypertension [12]. More than $90 \%$ of patients with an LSM $>20-25 \mathrm{kPa}$ ( evaluated by transient elastography ) will have clinically significant portal hypertension. In advanced chronic liver disease of non-cholestatic aetiology, endoscopy can be safety avoided by using LSM and platelet count in combination: LSM of $<20 \mathrm{kPa}$ and PLT $>150 \mathrm{~g} / \mathrm{L}$ pointed to $<5 \%$ risk of esophageal varices needing treatment [12].

Transient elastography (TE) (FibroScan, Echosens, France) is currently the most widely used technique, validated in chronic hepatitis $C$ [13], in primary biliary cholangitis (PBC) $[14,15]$ and primary sclerosing cholangitis [16]. TE measures the speed of propagation of an elastic shear wave in the liver, and the harder the tissue, the faster the shear, which is measured in kilopascals $(\mathrm{kPa})$. The examination is performed on the right lobe of the liver, and the measurement depth trough intercostal space is $25-65 \mathrm{~mm}$ using standard M-probe, and 35-75 mm with XL-probe (Figure 1). Liver stiffness measurement based on TE has been shown to correlate with histological fibrosis stage and severity of portal hypertension [17, 18]. TE seems to be a predictor of clinical outcomes in relationship to liverrelated complications and mortality $[19,20]$. Additionally, TE is able to predict clinically significant portal hypertension in patients with compensated chronic liver disease or cirrhosis [21]. However, early compensated liver cirrhosis can be overlooked in up to $30 \%$ of patients and transient elastography seems to be better at excluding advanced fibrosis rather than confirming liver cirrhosis. Fibrosis stage $\mathrm{F}>2$ is diagnosed with $84-87 \%$ accuracy, and F> 3 with $88-89 \%$. Diagnostic accuracy is excellent - 93-96\% for the diagnosis of liver cirrhosis, with sensitivity and specificity of $70-79 \%, 78-84 \%$ for $\mathrm{F}>2$ and $83-87 \%$ and 89-95\% for the diagnosis of $\mathrm{F}=4$. Cut-offs were in the range of 7.3-7.9 $\mathrm{kPa}$ for $\mathrm{F}>2$, and 13.0-15.6 kPa for the diagnosis of liver cirrhosis.

In the newest study of Krawczyk et al. TE correlated with Laennec stages of fibrosis, collagen contents and with diameter of thickest septa in explanted livers in PSC patients. In multivariate model liver fibrosis according to either Leannec score or collagen contents was significantly associated with TE. PSC cirrhotics patients had increased liver stiffness and the TE cut-off of $13.7 \mathrm{kPa}$ showed the best predictive value $(\mathrm{AUC}=0.90,95 \% \mathrm{CI} 0.80-1.00, \mathrm{P}<0.0001)$ for detecting liver cirrhosis [57].

The measurement failure rate is low (5-10\%) with obesity (BMI > $30 \mathrm{~kg} / \mathrm{m}^{2}$ ), ascites, congestive heart failure, postprandial time and the presence of narrow intercostal space considered to be limiting factors. However, obstructive cholestasis also influenced the results of TE [22].

Newer elastography methods based on the measurements of shear wave velocity include point share wave elastography (pSWE) and two-dimensional SWE (2D- SWE). SWE is usually integrated into conventional ultrasonography system (Figure 2). The region of interest (ROI) can be positioned under brightness-modulation (B-mode), and a single acoustic impulse is used to induce a share wave within a ROI of $1.0 \times 0.5 \mathrm{~cm}$ or $2 \times 2 \mathrm{~cm}$ in $2 \mathrm{D}$ SWE. The examination should be performed at least $1 \mathrm{~cm}$ below the liver capsule on the right lobe, and can be displayed in $\mathrm{m} / \mathrm{s}$ and/or $\mathrm{kPa}$. ROI can be positioned manually in different depths of the liver. However, there are no clear interpretation of point SWE and 2D SWE recommended to date.
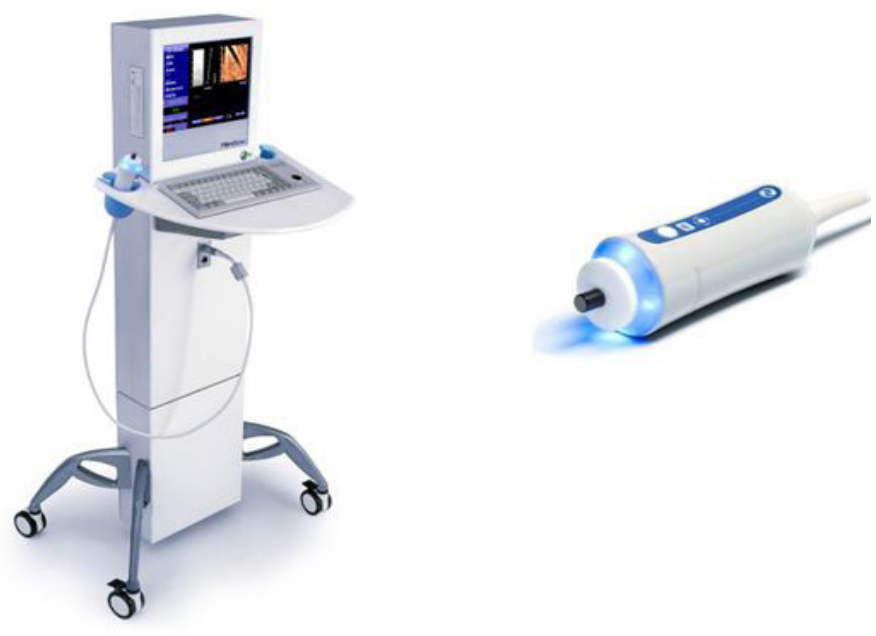

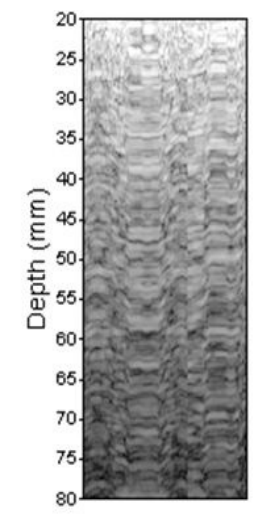

(a)

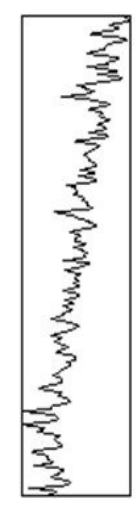

(b)

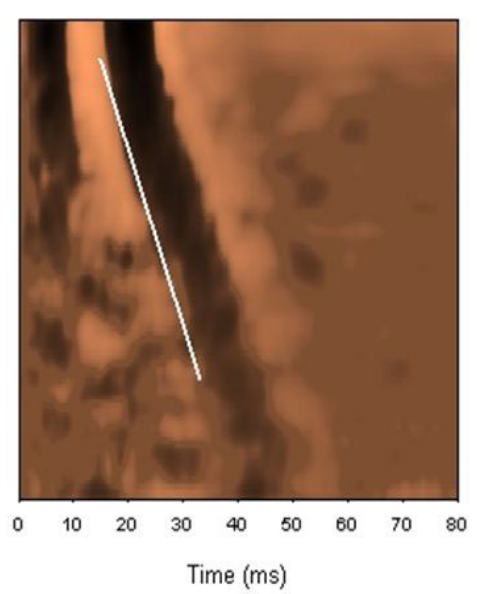

(c)
Figure 1. Transient elastrography.

The probability of correctly diagnosing EV following a positive measurement did not exceed 70\% [21]. Thus, LSM-spleen diameter to platelet ratio score and simplified combination of LSM and platelet count were also assessed with good results of ruling out varices needing treatment $[23,24]$. LSM can be also used to predict clinical decompensation in the patients with compensated cirrhosis of the liver. On the other hand, spleen undergoes parenchymal modeling in patients with portal hypertension, and spleen stiffness measurement (SSM) is closely associated with portal hypertension, its severity and complications [25]. SSM is promising parameter for use in predicting the presence and size of EV [12]. Validated cut-off values in PSC are not available yet.

\section{Magnetic-resonance based method}

With magnetic resonance elastography (MRE) mechanical shear waves are sent into the tissue and displayed as elastograms using phase-contrast image sequences. MRE can examine the very large areas of the right lobe of liver. The limitation of MRE are obesity, 

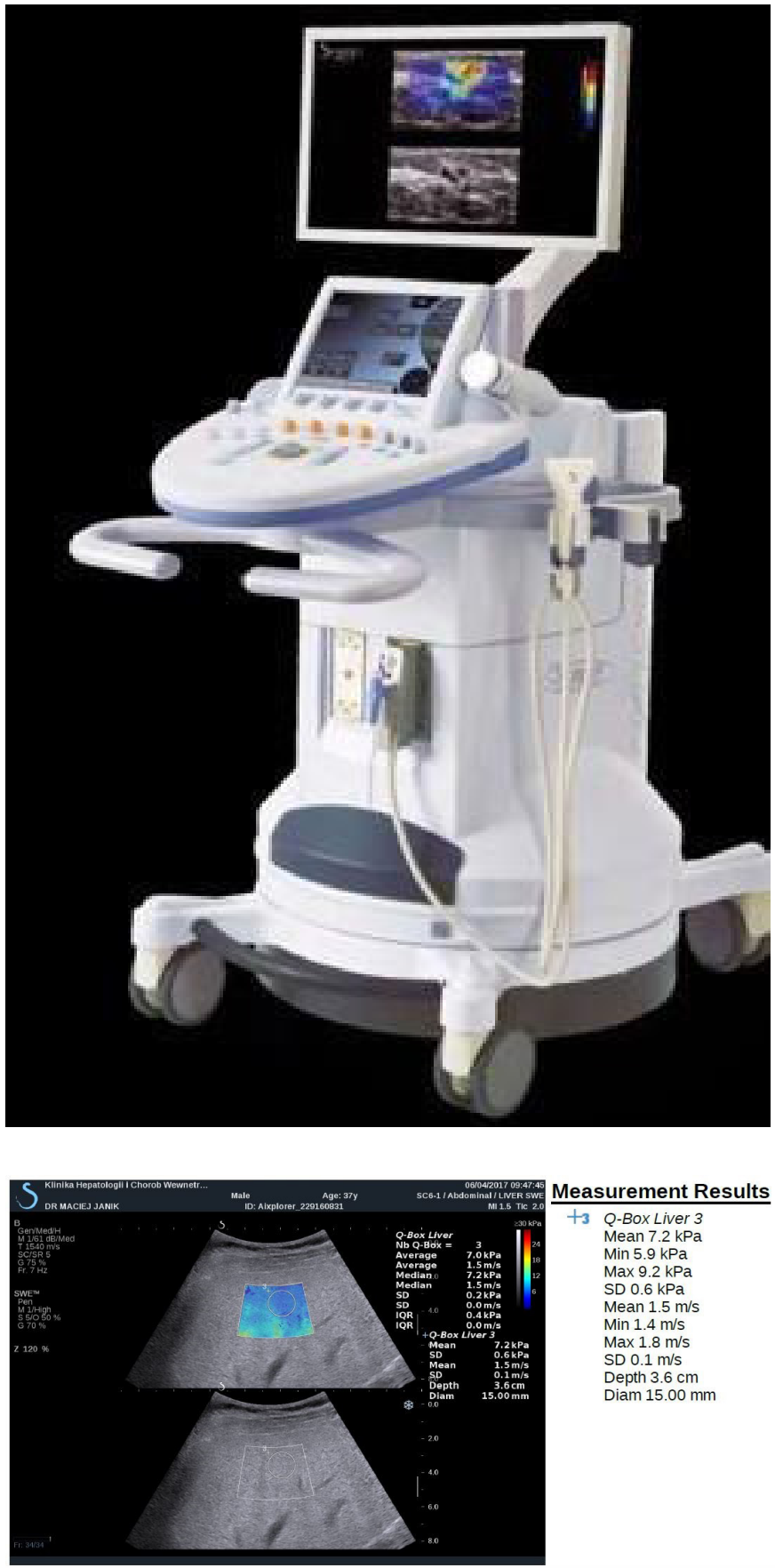

Figure 2. Shear-wave elastography.

claustrophobia and iron overload. Recently, in the study of Wang et al. the performance of MRE was significantly better than laboratory tests for detection of advanced fibrosis, and cirrhosis and better than conventional MRI for diagnosis of cirrhosis in patients with autoimmune hepatitis [26]. In a retrospective review of 266 PSC patients to examine whether liver stiffness (LS) was associated with the primary endpoint of hepatic decompensation (ascites, variceal hemorrhage and hepatic encephalopathy), MRE was able to detect cirrhosis with high specificity and LS obtained by MRE was predictive of hepatic decompensation in PSC patients in Eaton et al study. Liver stiffness of $4.93 \mathrm{kPa}$ was the optimal point to detected $\mathrm{F} 4$ fibrosis, with sensitivity 1.00 (95\% confidence interval (CI), 0.40-1.00) and specificity of 0.94 ( $95 \% \mathrm{CI}, 0.68-1.00)$. LS was associated with the development of decompensated liver disease (Hazard ratio, 1.55; 95\%CI, 1.41-1.70). The optimal LS thresholds that stratified patients at a low, medium and high risk for hepatic decompensation were $<4.5,4.5-6.0$ and $>6.0 \mathrm{kPa}$, respectively [27]. However, MRE seems to be promising modality for detection of advanced fibrosis and liver cirrhosis, with superior diagnostic accuracy compared to laboratory assessment and MRI, but not precirrhotic stages of chronic liver diseases. On the other hand, MRE is very expensive and time-consuming.

\section{Serum biomarkers}

Prospective studies demonstrated that single markers e. g., a2macroglobulin [28], procollagen III N-peptide [29], apolipoprotein A1 [28], haptoglobin [30], hyaluronic acid [31], metalloproteinases [32] allow discrimination between advanced and absent fibrosis.

The enhanced liver fibrosis (ELF) test is a promising panel, incorporating three direct serum markers of fibrosis in an algorithm: hyaluronic acid, tissue inhibitor of metalloproteinases-1 (TIMP-1), and amino-terminal pro-peptide of type III pro-collagen (PIINP) [33]. The ELF test accurately predicted significant liver fibrosis and furthermore predicted clinical outcome in several independent populations and in patients with various aetiologies of chronic liver disease [34] as well as with PSC. The ELF test consistently predicted liver transplant-free survival in PSC patients independently of other risk factors or risk scores [35]. The ELF test distinguished between mild and severe disease defined by clinical outcome (transplantation or death) with an area under the curve of 0.81 (95\% confidence interval [CI] 0.73-0.87) and optimal cutoff of 10.6 (sensitivity $70.2 \%$, specificity $79.1 \%$ ). In multivariate Cox regression analysis ELF score was associated with transplant-free survival independently of the Mayo risk score. The ELF test correlated also with ultrasound elastography in separate assessments [35]. In a large multicenter cohort, EFL test predicts prognosis in PSC and may be used for risk stratification in clinical follow up; optimally together with clinical prognostic scores may add incremental prognostic value [36].

Placental growth factor (PLGF), growth differentiation factor-15 (GDF-15) and hepatic growth factor (HGF) are involved in hepatic fibrogenesis. The panel of these three serum markers was useful for the detection of patients with advanced fibrosis and the risks described by the combinations of these markers were independent from other classical fibrosis risk factors. The set of markers may be a useful tool to monitor patients with chronic liver diseases during and after therapy [37] .

Inflammatory protein, i.e. IL-8 in bile and serum was an important indicator of disease severity and prognosis in patients with primary sclerosing cholangitis, and associated with transplant-free survival in multivariable analyses independently of age and disease duration, indicating an independent influence on PSC progression [38]. This is also in line with the results of the study of Buck et al [39]. Hepatic venous pressure gradient (HVPG) can reflect progression of disease in the precirrhosis stage. Portal hypertension is pathogenically related to liver injury and fibrosis [40] and that in turn these are associated with 
the activation of inflammatory pathways [41]. The novel inflammatory serum biomarkers (e.g. Il-1b, Fas-R, VCAM, CD163) were significantly correlated with HVPG in patients with compensated cirrhosis in this study.

Autotaxin (ATX), which is involved in the synthesis of lysophosphatidic acid, is not only associated with pruritus but also indicates impairment of other health-related quality of life (HRQoL) aspects, liver dysfunction, and can serve as a predictor of survival [42]. Impairment of HRQoL might be also associated with vitamin D receptor (VDR) gene polymorphisms (rs1544410-BsmI; rs7975232ApaI). ApaI polymorphisms in VDR may exert an effect on diseaserelated symptoms and quality of life in the study of 275 patients with PSC [43].

However, none of the proposed markers or panels have gained as much acceptance as the invasive approach [44]. This may be due to relatively high costs of marker measurements, and low sensitivity to discriminate between fibrotic, cirrhotic or steatotic liver lesions. As a result, no scores based on serum levels of hepatic fibrosis markers are actually regarded as definite methods upon which therapeutic decisions can be based. It might be that the combination of markers reflects the presence of significant liver fibrosis detected by elastography and histology and may also identify patients at risk presenting with low elastography values as proofed by Krawczyk M, et al. [37].

\section{Simple laboratory tests}

Laboratory-based methods for staging liver fibrosis include the FibroTest ${ }^{\circ}$ [45], the serum aspartate aminotransferase/platelet ratio index (APRI) [46], the Fibrosis 4 (FIB-4) test [47], and the enhanced liver fibrosis test [48]. AST/ALT ratio [49] can also allow to discriminate between advanced and absent fibrosis.

However, these tests may detect cirrhosis, but their ability to reflect the stages of fibrosis in AIH is uncertain [50-54]. The result of the recent study of Anastasiou et al. showed that TE, NAFLD fibrosis score and FibroQ might help in evaluation of liver fibrosis in AIH, but without differentiating mild form from advanced stages of fibrosis in autoimmune hepatitis [55].

In the study of Krawczyk et al. TE correlated with Laennec stages of fibrosis, and with serum indices of liver injury, namely AST, bilirubin as well as FIB-4 and APRI scores in patients with PSC [57].

\section{Conclusion}

Primary sclerosing cholangitis (PSC) is a progressive biliary disease lacking medical treatment with currently no established tools to predict prognosis in the individual patient. The lack of biomarkers for risk stratification is an important obstacle to the development of therapy.

Liver fibrosis seems to be the strongest predictor of liver stiffness assessed with TE. TE correlates with liver fibrosis, markers of liver injury and portal hypertension in patients with PSC. It might be that $\mathrm{TE}$ is a reliable tool for non-invasive monitoring of PSC. It seems also that the combination of serum profibrotic biomarkers with evaluation of liver fibrosis with elastography may improve the non-invasive diagnostic utility for clinically significant fibrosis [56]. However, still the Enhanced Liver Fibrosis $\left(\mathrm{ELF}^{\bullet}\right)$ test and Mayo risk score proved to be stronger predictors of transplant-free survival in PSC [38].

Conflict of interest: Nothing to declare

\section{References}

1. Weismuller TJ, Trivedi PJ, Bergquist A, Imam M, Lenzen H, Ponsioen CY, et al. (2017) Patient Age, Sex, and Inflammatory Bowel Disease Phenotype Associate With Course of Primary Sclerosing Cholangitis. Gastroenterology 152: 1975-84 e8.

2. Dickson ER, Murtaugh PA, Wiesner RH, Grambsch PM, Fleming TR, Ludwig J, et al. (1992) Primary sclerosing cholangitis: refinement and validation of survival models. Gastroenterology 103: 1893-901.

3. Kim G, Lee SS, Baik SK, Cho YZ, Kim MY, Kwon SO, et al. (2016) The need for histological subclassification of cirrhosis: a systematic review and meta-analysis. Liver Int 36: 847-55.

4. Kim SU1, Oh HJ, Wanless IR, Lee S, Han KH, et al. (2012) The Laennec staging system for histological sub-classification of cirrhosis is useful for stratification of prognosis in patients with liver cirrhosis. J Hepatol 57: 556-563. [crossref]

5. Rastogi A, Maiwall R, Bihari C, Ahuja A, Kumar A, Singh T, et al. (2013) Cirrhosis histology and Laennec staging system correlate with high portal pressure. Histopathology 62: 731-41

6. Wang W, Li J, Pan R, A S, Liao C (2015) Association of the Laennec staging system with degree of cirrhosis, clinical stage and liver function. Hepatol Int 9: 621-6.

7. La Mura V, Nicolini A, Tosetti G, Primignani M (2015) Cirrhosis and portal hypertension: The importance of risk stratification, the role of hepatic venous pressure gradient measurement. World J Hepatol 7: 688-95.

8. Garcia-Tsao G, Friedman S, Iredale J, Pinzani M (2010) Now there are many (stages) where before there was one: In search of a pathophysiological classification of cirrhosis. Hepatology 51: 1445-9.

9. Tsochatzis E, Bruno S, Isgro G, Hall A, Theocharidou E, Manousou P, et al. (2014) Collagen proportionate area is superior to other histological methods for subclassifying cirrhosis and determining prognosis. J Hepatol 60: 948-54.

10. Rockey DC, Caldwell SH, Goodman ZD, Nelson RC, Smith AD; American Association for the Study of Liver Diseases (2009) Liver biopsy. Hepatology 49: 1017-1044. [crossref]

11. Dietrich CF, Bamber J, Berzigotti A, Bota S, Cantisani V, Castera L, et al. (2017) EFSUMB Guidelines and Recommendations on the Clinical Use of Liver Ultrasound Elastography, Update 2017 (Long Version). Ultraschall Med.

12. Berzigotti A(2017) Non-invasive evaluation of portal hypertension using ultrasound elastography. J Hepatol 67: 399-411. [crossref]

13. Ziol M, Handra-Luca A, Kettaneh A, Christidis C, Mal F, et al. (2005) Noninvasive assessment of liver fibrosis by measurement of stiffness in patients with chronic hepatitis C. Hepatology 41: 48-54. [crossref]

14. Corpechot C, Carrat F, Poujol-Robert A, Gaouar F, Wendum D, Chazouilleres O, et al. (2012) Noninvasive elastography-based assessment of liver fibrosis progression and prognosis in primary biliary cirrhosis. Hepatology 56: 198-208.

15. Corpechot C1, El Naggar A, Poujol-Robert A, Ziol M, Wendum D, et al. (2006) Assessment of biliary fibrosis by transient elastography in patients with $\mathrm{PBC}$ and PSC. Hepatology 43: 1118-1124. [crossref]

16. Corpechot C, Gaouar F, El Naggar A, Kemgang A, Wendum D, Poupon R, et al. (2014) Baseline values and changes in liver stiffness measured by transient elastography are associated with severity of fibrosis and outcomes of patients with primary sclerosing cholangitis. Gastroenterology 146: 970-9; quiz e15-6.

17. Berzigotti A, Seijo S, Arena U, Abraldes JG, Vizzutti F, Garcia-Pagan JC, et al (2013) Elastography, spleen size, and platelet count identify portal hypertension in patients with compensated cirrhosis. Gastroenterology 144: 102-11 e1.

18. Talwalkar JA, Kurtz DM, Schoenleber SJ, West CP, Montori VM (2007) Ultrasound based transient elastography for the detection of hepatic fibrosis: systematic review and meta-analysis. Clin Gastroenterol Hepatol 5: 1214-20.

19. Vergniol J, Foucher J, Terrebonne E, Bernard PH, le Bail B, et al. (2011) Noninvasive tests for fibrosis and liver stiffness predict 5-year outcomes of patients with chronic hepatitis C. Gastroenterology 140: 1970-1979. [crossref]

20. Singh S, Fujii LL, Murad MH, Wang Z, Asrani SK, Ehman RL, et al. (2013) Liver stiffness is associated with risk of decompensation, liver cancer, and death in patients with chronic liver diseases: a systematic review and meta-analysis. Clin Gastroenterol Hepatol 11: 1573-84 e1-2; quiz e88-9.

21. Shi KQ, Fan YC, Pan ZZ, Lin XF, Liu WY, Chen YP, et al. (2013) Transient elastography: a meta-analysis of diagnostic accuracy in evaluation of portal hypertension in chronic liver disease. Liver Int 33: 62-71. 
22. Millonig G, Reimann FM, Friedrich S, Fonouni H, Mehrabi A, Buchler MW, et al. (2008) Extrahepatic cholestasis increases liver stiffness (FibroScan) irrespective of fibrosis. Hepatology 48: 1718-23.

23. Abraldes JG, Bureau C, Stefanescu H, Augustin S, Ney M, Blasco H, et al. (2016) Noninvasive tools and risk of clinically significant portal hypertension and varices in compensated cirrhosis: The "Anticipate" study. Hepatology 64: 2173-84.

24. Augustin S, Millan L, Gonzalez A, Martell M, Gelabert A, Segarra A, et al. (2014) Detection of early portal hypertension with routine data and liver stiffness in patients with asymptomatic liver disease: a prospective study. J Hepatol 60: 561-9.

25. Colecchia A, Montrone L, Scaioli E, Bacchi-Reggiani ML, Colli A, Casazza G, et al. (2012) Measurement of spleen stiffness to evaluate portal hypertension and the presence of esophageal varices in patients with HCV-related cirrhosis. Gastroenterology 143: 646-54.

26. Wang J, Malik N, Yin M, Smyrk TC, Czaja AJ, Ehman RL, et al. (2017) Magnetic resonance elastography is accurate in detecting advanced fibrosis in autoimmune hepatitis. World J Gastroenterol 23: 859-68.

27. Eaton JE, Dzyubak B, Venkatesh SK, Smyrk TC, Gores GJ, Ehman RL, et al. (2016) Performance of magnetic resonance elastography in primary sclerosing cholangitis. J Gastroenterol Hepatol 31: 1184-90.

28. Imbert-Bismut F, Ratziu V, Pieroni L, Charlotte F, Benhamou Y, Poynard T, et al. (2001) Biochemical markers of liver fibrosis in patients with hepatitis $C$ virus infection: a prospective study. Lancet 357: 1069-75.

29. Van Zanten RA, van Leeuwen RE, Wilson JH (1988) Serum procollagen III $\mathrm{N}$-terminal peptide and laminin P1 fragment concentrations in alcoholic liver disease and primary biliary cirrhosis. Clin Chim Acta 177: 141-6.

30. Adams LA, Bulsara M, Rossi E, DeBoer B, Speers D, George J, et al. (2005) Hepascore: an accurate validated predictor of liver fibrosis in chronic hepatitis $\mathrm{C}$ infection. Clin Chem 51: 1867-73.

31. Wong VS, Hughes V, Trull A, Wight DG, Petrik J, Alexander GJ (1998) Serum hyaluronic acid is a useful marker of liver fibrosis in chronic hepatitis $\mathrm{C}$ virus infection. $J$ Viral Hepat 5: 187-92.

32. Walsh KM, Timms P, Campbell S, MacSween RN, Morris AJ (1999) Plasma levels of matrix metalloproteinase-2 (MMP-2) and tissue inhibitors of metalloproteinases -1 and -2 (TIMP-1 and TIMP-2) as noninvasive markers of liver disease in chronic hepatitis C: comparison using ROC analysis. Dig Dis Sci 44: 624-30.

33. Xie Q, Zhou X, Huang P, Wei J, Wang W, et al. (2014) The performance of enhanced liver fibrosis (ELF) test for the staging of liver fibrosis: a meta-analysis. PLoS One 9: e92772. [crossref]

34. Parkes J, Roderick P, Harris S, Day C, Mutimer D, et al. (2010) Enhanced liver fibrosis test can predict clinical outcomes in patients with chronic liver disease. Gut 59: 1245-1251. [crossref]

35. Vesterhus M, Hov JR, Holm A, Schrumpf E, Nygard S, Godang K, et al. (2015) Enhanced liver fibrosis score predicts transplant-free survival in primary sclerosing cholangitis. Hepatology 62: 188-97.

36. de Vries EM, Farkkila M, Milkiewicz P, Hov JR, Eksteen B, Thorburn D, et al. (2017) Enhanced liver fibrosis test predicts transplant-free survival in primary sclerosing cholangitis, a multi-centre study. Liver Int.

37. Krawczyk M, Zimmermann S, Hess G, Holz R, Dauer M, Raedle J, et al. (2017) Panel of three novel serum markers predicts liver stiffness and fibrosis stages in patients with chronic liver disease. PLoS One 12: e0173506.

38. Vesterhus M, Holm A, Hov JR, Nygard S, Schrumpf E, Melum E, et al. (2017) Novel serum and bile protein markers predict primary sclerosing cholangitis disease severity and prognosis. J Hepatol 66: 1214-22.

39. Buck M, Garcia-Tsao G, Groszmann RJ, Stalling C, Grace ND, Burroughs AK, et al. (2014) Novel inflammatory biomarkers of portal pressure in compensated cirrhosis patients. Hepatology 59: 1052-9.

40. Bellot P, Garcia-Pagan JC, Frances R, Abraldes JG, Navasa M, Perez-Mateo M, et al. (2010) Bacterial DNA translocation is associated with systemic circulatory abnormalities and intrahepatic endothelial dysfunction in patients with cirrhosis. Hepatology 52: 2044-52.

41. Frances R, Rodriguez E, Munoz C, Zapater P, De la ML, Ndongo M, et al. (2005) Intracellular cytokine expression in peritoneal monocyte/macrophages obtained from patients with cirrhosis and presence of bacterial DNA. Eur J Gastroenterol Hepatol 17: 45-51.

42. Wunsch E, Krawczyk M, Milkiewicz M, Trottier J, et al. (2016) Serum Autotaxin is a Marker of the Severity of Liver Injury and Overall Survival in Patients with Cholestatic Liver Diseases. Sci Rep 6: 30847. [crossref]

43. Kempinska-Podhorodecka A, Milkiewicz M, Jablonski D, Milkiewicz P, Wunsch E (2017) ApaI polymorphism of vitamin D receptor affects health-related quality of life in patients with primary sclerosing cholangitis. PLoS One 12: e0176264

44. Poynard T (2011) First-line assessment of patients with chronic liver disease with non-invasive techniques and without recourse to liver biopsy. J Hepatol 54: 586-7.

45. Poynard T, Imbert-Bismut F, Ratziu V, Chevret S, Jardel C, Moussalli J, et al. (2002) Biochemical markers of liver fibrosis in patients infected by hepatitis C virus: longitudinal validation in a randomized trial. J Viral Hepat 9: 128-33.
46. Angulo P, Bugianesi E, Bjornsson ES, Charatcharoenwitthaya P, Mills PR, Barrera F, et al. (2013) Simple noninvasive systems predict long-term outcomes of patients with nonalcoholic fatty liver disease. Gastroenterology 145: 782-9 e4.

47. Shah AG, Lydecker A, Murray K, Tetri BN, Contos MJ, et al. (2009) Comparison of noninvasive markers of fibrosis in patients with nonalcoholic fatty liver disease. Clin Gastroenterol Hepatol 7: 1104-1112. [crossref]

48. Parkes J, Guha IN, Roderick P, Harris S, Cross R, Manos MM, et al. (2011) Enhanced Liver Fibrosis (ELF) test accurately identifies liver fibrosis in patients with chronic hepatitis C. J Viral Hepat 18: 23-31.

49. Sheth SG, Flamm SL, Gordon FD, Chopra S (1998) AST/ALT ratio predicts cirrhosis in patients with chronic hepatitis C virus infection. Am J Gastroenterol 93: 44-48. [crossref]

50. Gutkowski K, Hartleb M, Kacperek-Hartleb T, Kajor M, Mazur W, Zych W, et al. (2013) Laboratory-based scoring system for prediction of hepatic inflammatory activity in patients with autoimmune hepatitis. Liver Int 33: 1370-7.

51. Abdo AA (2006) Clinical presentation, response to therapy, and predictors of fibrosis in patients with autoimmune hepatitis in Saudi Arabia. Saudi J Gastroenterol 12: 73-76. [crossref]

52. Abdollahi M, Pouri A, Ghojazadeh M, Estakhri R, Somi M (2015) Non-invasive serum fibrosis markers: A study in chronic hepatitis. Bioimpacts 5: 17-23. [crossref]

53. Anastasiou J, Alisa A, Virtue S, Portmann B, Murray-Lyon I, Williams R (2010) Noninvasive markers of fibrosis and inflammation in clinical practice: prospective comparison with liver biopsy. Eur J Gastroenterol Hepatol 22: 474-80.

54. Luth S, Herkel J, Kanzler S, Frenzel C, Galle PR, Dienes HP, et al. (2008) Serologic markers compared with liver biopsy for monitoring disease activity in autoimmune hepatitis. J Clin Gastroenterol 42: 926-30.

55. O EA, Buchter M, H AB, Korth J, Canbay A, Gerken G, et al. (2016) Performance and Utility of Transient Elastography and Non-Invasive Markers of Liver Fiibrosis in Patients with Autoimmune Hepatitis: A Single Centre Experience. Hepat Mon 16: e40737.

56. Friedrich-Rust M, Poynard T, Castera L (2016) Critical comparison of elastography methods to assess chronic liver disease. Nat Rev Gastroenterol Hepatol 13: 402411. [crossref]

57. Marcin Krawczyk, Joanna Ligocka, Mariusz Ligocki, Joanna RaszejaWyszomirska, Małgorzata Milkiewicz, et al. (2017) Does transient elastography correlate with liver fibrosis in patients with PSC? Laennec score-based analysis of explanted livers DOI: 10.1080/00365521.2017.1370009

Citation:

Monika Szydlowska-Jakimiuk, Joanna Raszeja-Wyszomirska (2017) Surrogate Markers of Liver Fibrosis in Primary Sclerosing Cholangitis (PSC). Internal Med Res Open J Volume 2(3): 1-5 